\title{
Single Realization Stochastic FDTD for Weak Scattering Waves in Biological Random Media
}

\author{
Tengmeng Tan, Member, IEEE, Allen Taflove, Fellow, IEEE, and Vadim Backman
}

\begin{abstract}
This paper introduces an iterative scheme to overcome the unresolved issues presented in S-FDTD (stochastic finite-difference time-domain) for obtaining ensemble average field values recently reported by Smith and Furse in an attempt to replace the brute force multiple-realization also known as Monte-Carlo approach with a single-realization scheme. Our formulation is particularly useful for studying light interactions with biological cells and tissues having sub-wavelength scale features. Numerical results demonstrate that such a small scale variation can be effectively modeled with a random medium problem which when simulated with the proposed S-FDTD indeed produces a very accurate result.
\end{abstract}

Index Terms-Biological cells and tissues, FDTD, iterative, PDE, random media, S-FDTD, S-PDE, statistical fluctuations, sub-wavelength.

\section{INTRODUCTION}

B IOLOGICAL cells/tissues are an extremely complicated material and light interactions in this medium are therefore too complex to be solved using a deterministic formulation. For example, in additional to the fact that the heterogeneities of these materials are below the sub-wavelength scale, an exact knowledge of these heterogeneity variations is far from complete. A more useful formulation for such a small scale variation may come from a random medium problem. Rayleigh scattering of sunlight in the atmosphere causing diffuse sky radiation is such a great example of a random medium problem.

A brute force technique for evaluating a random medium problem is to run Monte-Carlo simulations with multiple realizations. In this implementation the statistical heterogeneities characterizing the random medium magnetic $\mu$ and electric $\epsilon$ and $\sigma$ are a priori. The Monte-Carlo simulation uses this statistical information to assemble a large number of realizations. To this end, field quantities of interest are the scattered fields and the ensemble average of these fields are computed by taking the arithmetic mean over the total number of realizations. Note

Manuscript received January 29, 2012; revised May 29, 2012; accepted July 17, 2012. Date of publication September 21, 2012; date of current version January 30,2013 . This work was supported by NSERC of Canada, NIH grants R01CA128641, R01 EB003682 and NSF grant CBET-0937987 of the USA.

T. Tan and V. Backman are with the Department of Biomedical Engineering, Robert R. McCormick School of Engineering and Applied Science, Northwestern University, Evanston, IL 60208-3107 USA (e-mail: tmtan.ong@gmail.com).

A. Taflove is with the Department of Electrical Engineering and Computer Science, Robert R. McCormick School of Engineering and Applied Science, Northwestern University, Evanston, IL 60208-3118.

Color versions of one or more of the figures in this paper are available online at http://ieeexplore.ieee.org.

Digital Object Identifier 10.1109/TAP.2012.2220105 that in this multiple-realization Monte-Carlo approach the exact number of realizations needed to converge to a true ensemble average is not yet known and therefore becomes a part of the approximation. Depending on the nature of its statistical correlation, a random medium problem may require tens or hundreds of thousands of realizations. This means a brute force approach is very inefficient, particularly for a $2 \mathrm{~d} / 3 \mathrm{~d}$ problem.

An efficient alternative is to formulate the problem in such a way that its ensemble averages are ready to be run in a single realization scheme. Early reports on running a single-realization Monte-Carlo relevant to numerical electromagnetic waves seem to appear in [1]-[4]. In [1], [2] the authors focused on electromagnetic field uncertainties as a consequence of statistical uncertainties caused by either material inhomogeneities, or fabrication and placement tolerances. These uncertainties are becoming important in modern electronic devices where the wavelength is commensurable to its physical size. Using a single-realization scheme to evaluate radiation absorption in the human body affected by uncertainty measurements in tissues was reported in [3], [4]. The authors in this paper called the technique stochastic or S-FDTD, because the governing equations were taken directly from Maxwell's equations discretized with FDTD (finite-difference time-domain) methodology. On the other hand, the governing electromagnetic equations in [1] are taken from transmission-line matrix or TLM formulation.

In both cases their ensemble averages are constructed by evoking the definition of expectation operator applied directly to the governing numerical schemes updating the wave propagations. However, due to the fact that fluctuations in material quantities $\mu, \epsilon$, and $\sigma$ are directly coupled to the electric $\mathbf{E}$ and magnetic $\mathbf{H}$, that is $\epsilon \partial_{t} \mathbf{E}, \sigma \mathbf{E}$, and $\mu \partial_{t} \mathbf{H}$ where $\partial_{t}$ denote partial derivative with respect to time $t$, Taylor expansions were needed to further approximate these coupling terms. As a consequence, there are correlation coefficients that need to be resolved. It should be pointed out that such a coupling difficulty in mathematical literature is known as a multiplicative noise, and this type of noise in general is very difficult to solve [5], [6]. A unique procedure to relate the truncation error in Taylor expansion to that of the statistical moments that results in an optimized solution having significantly fewer numbers of realizations was also developed in [7].

In this paper we are proposing an iterative technique which will reformulate the multiplicative noise into an additive noise. Additive noise in Lagenvin's words is "infinitely simpler" to solve [8]. In this scheme the accuracy is determined by the number of the iterations which for a weak scattering problem can be as small as two or three iterations. To this end, the FDTD (finite-difference time-domain) algorithm established 
by Yee [9] has proven to be one of the most robust numerical tools to solve wave propagations including complex media exhibiting high levels of heterogeneities [10]-[12]. The method directly discretizes Maxwell's equations, and hence it contains the true physics of classical electrodynamics. This means that FDTD is fully equipped for studying wave propagation in random media. Following [3], [4], we also refer to this random medium single realization formulation as S-FDTD, because the numerical implementation also continues with the standard Yee's algorithm. The formulation of the method is presented in Section II, which will include discussions on multiplicative and additive noise. Section III provides numerical examples of the technique in practice, which is followed by discussion and conclusion.

\section{FORMULATION}

Let $\mathbf{E}_{T_{m}}(\mathbf{x}, t)$ and $\mathbf{H}_{T_{m}}(\mathbf{x}, t)$ represent the total electric and magnetic fields with $\mathbf{x}=(x, y, z)$ denoting spatial coordinate and $t$ denoting time. Maxwell's equations for waves propagating in a heterogeneous or random medium sourced by a temporal excitation $\mathbf{f}\left(\mathbf{x}_{o}, t\right)$ at a point $\mathbf{x}_{o}$ can be written as

$$
\begin{aligned}
& \epsilon_{m}(\mathbf{x}) \partial_{t} \mathbf{E}_{T_{m}}(\mathbf{x}, t)+\sigma_{m}(\mathbf{x}) \mathbf{E}_{T_{m}}(\mathbf{x}, t)=\nabla \times \mathbf{H}_{T_{m}}(\mathbf{x}, t) \\
& -\mu_{m}(\mathbf{x}) \partial_{t} \mathbf{H}_{T_{m}}(\mathbf{x}, t)=\nabla \times \mathbf{E}_{T_{m}}(\mathbf{x}, t)+\mathbf{f}\left(\mathbf{x}_{o}, t\right)
\end{aligned}
$$

The subscript $m$ is an integer signifying a specific realization generated by one particular sample associated with the known statistical distributions $\epsilon_{m}(\mathbf{x}), \sigma_{m}(\mathbf{x})$, and $\mu_{m}(\mathbf{x})$. For example, in a multiple-realization Monte-Carlo approach, the idea is to generate as many realizations as required (a large $m$ value) so that ensemble averages can be approximated from such a set of simulations.

If one associates a non-dispersive electric medium $\epsilon_{B}(\mathbf{x})$ and $\sigma_{B}(\mathbf{x})$ and magnetic medium $\mu_{B}(\mathbf{x})$ to be the average electromagnetic medium which by definition do not change over any realization $m$ then a wave propagating in this effective average medium is known to satisfy the incident fields

$$
\begin{gathered}
\epsilon_{B}(\mathbf{x}) \partial_{t} \mathbf{E}_{\mathrm{inc}}(\mathbf{x}, t)+\sigma_{B}(\mathbf{x}) \mathbf{E}_{\mathrm{inc}}(\mathbf{x}, t)=\nabla \times \mathbf{H}_{\mathrm{inc}}(\mathbf{x}, t) \\
-\mu_{B}(\mathbf{x}) \partial_{t} \mathbf{H}_{\mathrm{inc}}(\mathbf{x}, t)=\nabla \times \mathbf{E}_{\mathrm{inc}}(\mathbf{x}, t)+\mathbf{f}\left(\mathbf{x}_{o}, t\right)
\end{gathered}
$$

Detailed physical discussions on the existence of such an averaging process derived from the microscopic viewpoints can be found in Jackson [13]. The subscript letter $B$ is to emphasize background materials which are not fluctuating with $m$ but can vary with $x$.

Next, the total fields are partitioned into incident and scattered fields $\mathbf{E}_{T_{m}}=\mathbf{E}_{\text {inc }}+\mathbf{E}_{S_{m}}$ and $\mathbf{H}_{T_{m}}=\mathbf{H}_{\text {inc }}+\mathbf{H}_{S_{m}}$. Substituting these partitions into (1) and then subtracting the incident wave using (2), it follows that the scattered field can be written as

$$
\begin{aligned}
& {\left[\epsilon_{B}(\mathbf{x})+\Delta \epsilon_{m}(\mathbf{x})\right] \partial_{t} \mathbf{E}_{S_{m}}(\mathbf{x}, t)+\left[\sigma_{B}(\mathbf{x})+\Delta \sigma_{m}(\mathbf{x})\right] \mathbf{E}_{S_{m}}(\mathbf{x}, t)} \\
& \quad=\nabla \times \mathbf{H}_{S_{m}}(\mathbf{x}, t)-\Delta \epsilon_{m}(\mathbf{x}) \partial_{t} \mathbf{E}_{\mathrm{inc}}(\mathbf{x}, t) \\
& \quad-\Delta \sigma_{m}(\mathbf{x}) \mathbf{E}_{\mathrm{inc}}(\mathbf{x}, t) \\
& -\left[\mu_{B}(\mathbf{x})+\Delta \mu_{m}(\mathbf{x})\right] \partial_{t} \mathbf{H}_{S_{m}}(\mathbf{x}, t) \\
& \quad=\nabla \times \mathbf{E}_{S_{m}}(\mathbf{x}, t)+\Delta \mu_{m}(\mathbf{x}) \partial_{t} \mathbf{H}_{\mathrm{inc}}(\mathbf{x}, t) .
\end{aligned}
$$

Note that the fluctuations are defined as $\Delta \epsilon_{m}(\mathbf{x})=\epsilon_{m}(\mathbf{x})-$ $\epsilon_{B}(\mathbf{x})$ and $\Delta \mu_{m}(\mathbf{x})=\mu_{m}(\mathbf{x})-\mu_{B}(\mathbf{x})$. Equation (3) makes it very clear that a non-zero statistical uncertainty or fluctuation in $\Delta \epsilon_{m}, \Delta \sigma_{m}$ or $\Delta \mu_{m}$ will give rise to uncertainties in scattered fields $\mathbf{E}_{S_{m}}$ and $\mathbf{H}_{S_{m}}$. As a result, there will also be fluctuations in $\mathbf{E}_{T_{m}}$ and $\mathbf{H}_{T_{m}}$ because statistical fluctuations are superimposing the classical microscopic average incident fields $\mathbf{E}_{\text {inc }}$ and $\mathbf{H}_{\text {inc }}$. This is a well-known scattered field and total field decomposition formulation which can be found in many text books on electromagnetic theories [14]-[16]. Scattered fields can therefore be viewed as a consequence of electromagnetic materials deviating from their mean values. This is the central idea for constructing a weak scattering random medium problem.

\section{A. Multiplicative Noise}

In random medium problems executed with a multiple-realization Monte-Carlo approach, the quantities of interest are the ensemble average of the scattered fields. Mathematically, these averaging values are obtained via the discrete expectation operator summing over one single variable

$$
\begin{aligned}
\left\langle\mathbf{E}_{S}(\mathbf{x}, t)\right\rangle & =\lim _{M \rightarrow \infty} \frac{1}{M} \sum_{m=0}^{M-1} \mathbf{E}_{S_{m}}(\mathbf{x}, t) \\
\left\langle\mathbf{H}_{S}(\mathbf{x}, t)\right\rangle & =\lim _{M \rightarrow \infty} \sum_{m=0}^{M-1} \frac{1}{M} \mathbf{H}_{S_{m}}(\mathbf{x}, t) .
\end{aligned}
$$

An ensemble quantity therefore is a single value not depending on one particular realization $m$ but on the resulting average of all realization $m$. All other statistical average values are also defined in this way. In other words, the Monte-Carlo approach fundamentally assumes that a realization $m$ is simply one particular discrete sample whose infinite sum defines an ensemble average.

Applying such a discrete expectation directly to (3) gives rise to

$$
\begin{aligned}
& \left\langle\left[\epsilon_{B}(\mathbf{x})+\Delta \epsilon_{m}(\mathbf{x})\right] \partial_{t} \mathbf{E}_{S_{m}}(\mathbf{x}, t)\right\rangle+ \\
& \left\langle\left[\sigma_{B}(\mathbf{x})+\Delta \sigma_{m}(\mathbf{x})\right] \mathbf{E}_{S_{m}}(\mathbf{x}, t)\right\rangle=\nabla \times\left\langle\mathbf{H}_{S}(\mathbf{x}, t)\right\rangle+ \\
& -\langle\Delta \epsilon(\mathbf{x})\rangle \partial_{t} \mathbf{E}_{\mathrm{inc}}(\mathbf{x}, t)-\langle\Delta \sigma(\mathbf{x})\rangle \mathbf{E}_{\mathrm{inc}}(\mathbf{x}, t) \\
& \begin{array}{r}
\left.\left\langle\mu_{B}(\mathbf{x})+\Delta \mu_{m}(\mathbf{x})\right] \partial_{t} \mathbf{H}_{S_{m}}(\mathbf{x}, t)\right\rangle=-\nabla \times\left\langle\mathbf{E}_{S}(\mathbf{x}, t)\right\rangle \\
+\langle\Delta \mu(\mathbf{x})\rangle \partial_{t} \mathbf{H}_{\mathrm{inc}}(\mathbf{x}, t) .
\end{array}
\end{aligned}
$$

These equations need further clarifications. First notice that every ensemble term on the right-hand-side is already expressed as a single number which no longer depends on the realization $m$. This means that each of these terms is readily evaluated or replaced with one single variable. On the other hand, because all the terms on the left-hand-side of (3) are multiplications of two statistical variables which depend on $m$, it then follows that their ensemble averages need to be summed over the multiplications which in this case depends on the realization $m$. This multiplication relation is emphasized by the subscript $m$ inside the expectation as appeared in the left-hand-side of the ensemble (5). This notation will be used whenever there is a need to emphasize the difficulty of resolving multiplication of two statistical variables. 
The complication of achieving an algorithm that can be evaluated with a single-realization for a random medium problem is fundamentally rooted in the fact that both expectations $\left\langle\left[\epsilon_{B}(\mathbf{x})+\Delta \epsilon_{m}(\mathbf{x})\right] \partial_{t} \mathbf{E}_{S_{m}}(\mathbf{x}, t)\right\rangle$ and $\left\langle\left[\sigma_{B}(\mathbf{x})+\Delta \sigma_{m}(\mathbf{x})\right] \mathbf{E}_{S_{m}}(\mathbf{x}, t)\right\rangle$ have a coupled relation. That is, the input $\Delta \epsilon_{m}(\mathbf{x})$ and $\Delta \sigma_{m}(\mathbf{x})$ which depend on the realization $m$ are coupled to the output $\mathbf{E}_{S_{m}}(\mathbf{x}, t)$ which also depends on $m$. In stochastic partial differential equations (S-PDE), this coupling effect is called a multiplicative noise [5], [6], [8], in the sense that the input random variables $\Delta \epsilon_{m}$ and $\Delta \sigma_{m}$ and the output random variable $\mathbf{E}_{S_{m}}$ are related by a multiplication operation. This type of coupling effect is known to be a difficult S-PDE problem. Note that a non-magnetic material having $\mu_{m}(\mathbf{x})=\mu_{B}(\mathbf{x})=\mu_{o}$ or $\Delta \mu_{m}(\mathbf{x})=0$ for all $m$ reduces equation (5b) to

$$
-\mu_{o} \partial_{t}\left\langle\mathbf{H}_{S}(\mathbf{x}, t)\right\rangle=\nabla \times\left\langle\mathbf{E}_{S}(\mathbf{x}, t)\right\rangle .
$$

In a non-magnetic material the fluctuations in the scattered fields $\mathbf{E}_{S_{m}}$ and $\mathbf{H}_{S_{m}}$, or the total fields $\mathbf{E}_{T_{m}}=\mathbf{E}_{S_{m}}+\mathbf{E}_{\text {inc }}$ and $\mathbf{H}_{T_{m}}=\mathbf{H}_{S_{m}}+\mathbf{H}_{\text {inc }}$ are therefore strictly coming from the electrical fluctuations $\Delta \epsilon_{m}(\mathbf{x})$ and $\Delta \sigma_{m}(\mathbf{x})$. With no loss of generality, the remaining discussion in this paper assumes a nonmagnetic material $\Delta \mu_{m}(\mathbf{x})=0$ for all realization $m$ over any spatial $\mathbf{x}$.

The authors in both [1], [2] and [3], [4] make use of Taylor series expansions to approximate the coupled or multiplicativenoise terms. For example, it is possible to approximate the ensemble average of the coupled term for the scattered field on the left-hand-side of (5) with

$$
\begin{aligned}
& \left\langle\left[\epsilon_{B}(\mathbf{x})+\Delta \epsilon_{m}(\mathbf{x})\right] \partial_{t} \mathbf{E}_{S_{m}}(\mathbf{x}, t)\right\rangle \\
& \quad \approx \rho_{\epsilon}\left\langle\left[\epsilon_{B}(\mathbf{x})+\Delta \epsilon_{m}(\mathbf{x})\right]\right\rangle \partial_{t}\left\langle\mathbf{E}_{S_{m}}(\mathbf{x}, t)\right\rangle \\
& \left\langle\left[\sigma_{B}(\mathbf{x})+\Delta \sigma_{m}(\mathbf{x})\right] \mathbf{E}_{S_{m}}(\mathbf{x}, t)\right\rangle \\
& \quad \approx \rho_{\sigma}\left\langle\left[\sigma_{B}(\mathbf{x})+\Delta \sigma_{m}(\mathbf{x})\right]\right\rangle\left\langle\mathbf{E}_{S_{m}}(\mathbf{x}, t)\right\rangle
\end{aligned}
$$

where $\rho_{\epsilon}$ and $\rho_{\sigma}$ are correlation coefficients introduced to reduce numbers of realizations. That is to say, if these coefficients are known (accurately) then (5) effectively reduces to a deterministic wave propagation problem having an effective medium characterized by $\rho_{\epsilon}\left\langle\left[\epsilon_{B}(\mathbf{x})+\Delta \epsilon_{m}(\mathbf{x})\right]\right\rangle$ and $\rho_{\sigma}\left\langle\left[\sigma_{B}(\mathbf{x})+\Delta \sigma_{m}(\mathbf{x})\right]\right\rangle$. Again, the sum of the scattered and incident fields gives the needed total fields propagating within the interior and exterior of the medium.

\section{B. Additive Noise and Multiple Scattering}

We now introduce an iterative procedure to turn the multiplicative noise into an additive noise. Additive noise is known to be one of the simplest S-PDE to solve [5], [6], [8]. For example, the well-celebrated Lagenvin equation that motivated a systematic study of S-PDE is an additive noise model. To this end, we first partition the scattered fields into a superposition of linearly independent subset solutions

$$
\mathbf{E}_{S_{m}}=\sum_{q=1}^{Q} \mathbf{E}_{S_{m}}^{(q)} \quad \text { and } \quad \mathbf{H}_{S_{m}}=\sum_{q=1}^{Q} \mathbf{H}_{S_{m}}^{(q)} .
$$

Note that the superscript integer $q$ with the bracket is not meant to be an exponential. It is only a marker identifying their linear independency. It will be shown soon that $q$ is directly related to the order of accuracy. The corresponding ensemble averages are

$$
\left\langle\mathbf{E}_{S}\right\rangle=\sum_{q=1}^{Q}\left\langle\mathbf{E}_{S}^{(q)}\right\rangle \quad \text { and } \quad\left\langle\mathbf{H}_{S}\right\rangle=\sum_{q=1}^{Q}\left\langle\mathbf{H}_{S}^{(q)}\right\rangle .
$$

We next rewrite the scattered field (3a) as

$$
\begin{array}{r}
\epsilon_{B}(\mathbf{x}) \partial_{t} \mathbf{E}_{S_{m}}(\mathbf{x}, t)+\sigma_{B}(\mathbf{x}) \mathbf{E}_{S_{m}}(\mathbf{x}, t)=\nabla \times \mathbf{H}_{S_{m}}(\mathbf{x}, t)+ \\
-\Delta \epsilon_{m}(\mathbf{x}) \partial_{t}\left[\mathbf{E}_{\mathrm{inc}}(\mathbf{x}, t)+\mathbf{E}_{S_{m}}(\mathbf{x}, t)\right] \\
-\Delta \sigma_{m}(\mathbf{x})\left[\mathbf{E}_{\mathrm{inc}}(\mathbf{x}, t)+\mathbf{E}_{S_{m}}(\mathbf{x}, t)\right] .
\end{array}
$$

From the physical argument, it is clear that smaller statistical fluctuations $\Delta \epsilon_{m}$ and $\Delta \sigma_{m}$ result in a smaller scattered field as well. For example, if $\Delta \epsilon_{m}=\Delta \sigma_{m}=0$ then both statistical fluctuations $\mathbf{E}_{S_{m}}$ and $\mathbf{H}_{S_{m}}$ must be identically zero. Likewise, if these scattered fields are much smaller than the incident field then it is possible to approximate $\mathbf{E}_{\mathrm{inc}}+\mathbf{E}_{S_{m}} \approx \mathbf{E}_{\mathrm{inc}}$ and this error depends both on $\mathbf{E}_{\text {inc }}$ and $\mathbf{E}_{S_{m}}$. This means that the multiplicative noise in (10) to a first order accuracy (first iteration or guess) can be approximated with an additive noise. In particular, by assigning this first iteration to $\mathbf{E}_{S_{m}}^{(1)}$ and $\mathbf{H}_{S_{m}}^{(1)}$ the resulting additive noise S-PDE becomes

$$
\begin{gathered}
\epsilon_{B}(\mathbf{x}) \partial_{t} \mathbf{E}_{S_{m}}^{(1)}(\mathbf{x}, t)+\sigma_{B}(\mathbf{x}) \mathbf{E}_{S_{m}}^{(1)}(\mathbf{x}, t)=\nabla \times \mathbf{H}_{S_{m}}^{(1)}(\mathbf{x}, t)+ \\
-\Delta \epsilon_{m}(\mathbf{x}) \partial_{t} \mathbf{E}_{\mathrm{inc}}(\mathbf{x}, t)-\Delta \sigma_{m}(\mathbf{x}) \mathbf{E}_{\mathrm{inc}}(\mathbf{x}, t) \\
-\mu_{o} \partial_{t} \mathbf{H}_{S_{m}}^{(1)}(\mathbf{x}, t)=\nabla \times \mathbf{E}_{S_{m}}^{(1)}(\mathbf{x}, t) .
\end{gathered}
$$

Since $\mathbf{E}_{\text {inc }}(\mathbf{x}, t)$ is known for all necessary $\mathbf{x}$ and $t$, and independent of $m$, it follows that knowing the statistical $\Delta \epsilon_{m}(\mathbf{x})$ and $\Delta \sigma_{m}(\mathbf{x})$ the scattered fields $\mathbf{E}_{S_{m}}^{(1)}$ and $\mathbf{H}_{S_{m}}^{(1)}$ can be easily evaluated using any known PDE methodology. In other words, the first order approximation assumes the scattered fields are directly excited by the incident $\Delta \epsilon_{m}(\mathbf{x}) \partial_{t} \mathbf{E}_{\text {inc }}(\mathbf{x}, t)$ and $\Delta \sigma_{m}(\mathbf{x}) \mathbf{E}_{\text {inc }}(\mathbf{x}, t)$. It is therefore convenient to think of these excitations as a physical polarization current responsible for generating a scattered field.

The ensemble average variables $\left\langle\mathbf{E}_{S}^{(1)}\right\rangle$ and $\left\langle\mathbf{H}_{S}^{(1)}\right\rangle$ which are independent of the realization $m$ can now be evaluated using a single realization scheme. This is achieved by directly applying the discrete expectation to (11). Specifically,

$$
\begin{array}{r}
\epsilon_{B}(\mathbf{x}) \partial_{t}\left\langle\mathbf{E}_{S}^{(1)}(\mathbf{x}, t)\right\rangle+\sigma_{B}(\mathbf{x})\left\langle\mathbf{E}_{S}^{(1)}(\mathbf{x}, t)\right\rangle= \\
\nabla \times\left\langle\mathbf{H}_{S}^{(1)}(\mathbf{x}, t)\right\rangle+ \\
-\langle\Delta \epsilon(\mathbf{x})\rangle \partial_{t} \mathbf{E}_{\mathrm{inc}}(\mathbf{x}, t)-\langle\Delta \sigma(\mathbf{x})\rangle \mathbf{E}_{\mathrm{inc}}(\mathbf{x}, t) \\
-\mu_{o} \partial_{t}\left\langle\mathbf{H}_{S}^{(1)}(\mathbf{x}, t)\right\rangle=\nabla \times\left\langle\mathbf{E}_{S}^{(1)}(\mathbf{x}, t)\right\rangle .
\end{array}
$$

In this case the ensemble polarization currents are the incident $\langle\Delta \epsilon(\mathbf{x})\rangle \partial_{t} \mathbf{E}_{\text {inc }}(\mathbf{x}, t)$ and $\langle\Delta \sigma(\mathbf{x})\rangle \mathbf{E}_{\text {inc }}(\mathbf{x}, t)$. For completeness, we state that a zero statistical mean $\langle\Delta \epsilon(\mathbf{x})\rangle=0$ or $\langle\Delta \sigma(\mathbf{x})\rangle=0$ to a first order accuracy formulation produces 
no scattered field. Such a statistical distribution requires at least a second order iteration $q=2$. The question of what contributes as a first order accuracy solution (weak scattered field problem) in general will depend on both the statistical average $\langle\Delta \epsilon(\mathbf{x})\rangle$ and $\langle\Delta \sigma(\mathbf{x})\rangle$ and their spatial correlation length to be discussed in later sections. It will also be shown numerically that this accuracy also depends on the bandwidth of the incident excitation $\mathbf{f}\left(\mathbf{x}_{o}, t\right)$.

Substituting the linearly independent decomposition (8) into (10) results in a set of iteration equalities

$$
\begin{aligned}
\epsilon_{B}(\mathbf{x}) \partial_{t} \mathbf{E}_{S_{m}}^{(q+1)}(\mathbf{x}, t)+\sigma_{B}(\mathbf{x}) \mathbf{E}_{S_{m}}^{(q+1)}(\mathbf{x}, t) \\
=\nabla \times \mathbf{H}_{S_{m}}^{(q+1)}(\mathbf{x}, t)-\Delta \epsilon_{m}(\mathbf{x}) \partial_{t} \mathbf{E}_{S_{m}}^{(q)}(\mathbf{x}, t) \\
\quad-\Delta \sigma_{m}(\mathbf{x}) \mathbf{E}_{S_{m}}^{(q)}(\mathbf{x}, t) \\
-\mu_{o} \partial_{t} \mathbf{H}_{S_{m}}^{(q+1)}(\mathbf{x}, t)=\nabla \times \mathbf{E}_{S_{m}}^{(q+1)}(\mathbf{x}, t)
\end{aligned}
$$

with the understanding that $\mathbf{E}_{S_{m}}^{(0)}(\mathbf{x}, t)=\mathbf{E}_{\text {inc }}(\mathbf{x}, t)$. It is also true that if $\left|\Delta \epsilon_{m}(\mathbf{x})\right|$ is much smaller than $\epsilon_{B}(\mathbf{x})$ and $\left|\Delta \sigma_{m}(\mathbf{x})\right|$ smaller than $\sigma_{B}(\mathbf{x})$ then in general $\left|\mathbf{E}_{S_{m}}^{(q-1)}(\mathbf{x}, t)\right|>$ $\left|\mathbf{E}_{S_{m}}^{(q)}(\mathbf{x}, t)\right|$ and $\left|\mathbf{H}_{S_{m}}^{(q-1)}(\mathbf{x}, t)\right|>\left|\mathbf{H}_{S_{m}}^{(q)}(\mathbf{x}, t)\right|$. These are essentially the consequence of a weak scattering property where in each iteration (each of the multiple scattering) the scattered fields will be smaller than their excitations [17]. The implication is that the proposed methodology when applied to a weak scattering problem will converge quickly to a useful result after a few iterations - a smaller $q$. Lastly, note also that the polarization currents at each $q+1$ are simply the excitations $\Delta \epsilon_{m}(\mathbf{x}) \partial_{t} \mathbf{E}_{S_{m}}^{(q)}(\mathbf{x}, t)$ and $\Delta \sigma_{m}(\mathbf{x}) \mathbf{E}_{S_{m}}^{(q)}(\mathbf{x}, t)$.

Although every iteration in (13) now takes a form of an additive noise S-PDE, a single-realization scheme for $q>1$ is still not yet available. Specifically, the ensemble averages for the above iterative Maxwell's equations evaluate to

$$
\begin{array}{r}
\epsilon_{B}(\mathbf{x}) \partial_{t}\left\langle\mathbf{E}_{S}^{(q+1)}(\mathbf{x}, t)\right\rangle+\sigma_{B}(\mathbf{x})\left\langle\mathbf{E}_{S}^{(q+1)}(\mathbf{x}, t)\right\rangle= \\
\nabla \times\left\langle\mathbf{H}_{S}^{(q+1)}(\mathbf{x}, t)\right\rangle+ \\
-\left\langle\Delta \epsilon_{m}(\mathbf{x}) \partial_{t} \mathbf{E}_{S_{m}}^{(q)}(\mathbf{x}, t)\right\rangle-\left\langle\Delta \sigma_{m}(\mathbf{x}) \mathbf{E}_{S_{m}}^{(q)}(\mathbf{x}, t)\right\rangle \\
-\mu_{o} \partial_{t}\left\langle\mathbf{H}_{S}^{(q+1)}(\mathbf{x}, t)\right\rangle=\nabla \times\left\langle\mathbf{E}_{S}^{(q+1)}(\mathbf{x}, t)\right\rangle .
\end{array}
$$

The coupling or multiplicative noise difficulty now resides in the polarization currents $\Delta \epsilon_{m}(\mathbf{x}) \partial_{t} \mathbf{E}_{S_{m}}^{(q)}(\mathbf{x}, t)$ and $\Delta \sigma_{m}(\mathbf{x}) \mathbf{E}_{S_{m}}^{(q)}(\mathbf{x}, t)$. This can be resolved by explicitly expressing $\mathbf{E}_{S_{m}}^{(q)}(\mathbf{x}, t)$ in terms of the known deterministic incident $\mathbf{E}_{\text {inc }}(\mathbf{x}, t)$ and all other statistical ensembles. The process should start with the lowest iteration (11). For example, if a first order iteration $q=1$ is needed then solution to this iteration can be obtained in many different ways. However, if another higher order accuracy is needed then it may be necessary to first approximate the first order solution and then use the result to construct the second order iteration $q=2$. This process continues until an acceptable result is achieved.

To this end, constructing a solution for $\mathbf{E}_{S_{m}}^{(q)}(\mathbf{x}, t)$ to be expressed explicitly in terms of the known incident $\mathbf{E}_{\text {inc }}(\mathbf{x}, t)$ and all other statistical parameters follows a rather straightforward process. The challenge lies in the numerical efficiency. Specifically, it will be made clear that not making use of symmetry (or redundancy) will result in a numerical complexity reaching $N^{d \times q}$ with $N$ referring to discrete points in the random medium and $d$ the physical dimension of the problem. For example, if the details of a three dimension $d=3$ random medium numerically requires $N=5,000^{3}$ discrete resolution points, then a second order accuracy will have a complexity of $5,000^{6}$. This value could even be less efficient than running the multiple-realization Monte-Carlo scheme. It is therefore vitally important to make use of redundancy to reduce this computational complexity.

For a $1 \mathrm{~d}$ problem it is possible to reduce this complexity to linearly proportional to $q$. However, $1 \mathrm{~d}$ and $2 \mathrm{~d} / 3 \mathrm{~d}$ wave problems differ in a very fundamental way. For example, the Green's function for the 1d wave propagating in a homogeneous medium without boundary condition follows a very simple expression (has no singularity). The Green's function for a $2 \mathrm{~d} / 3 \mathrm{~d}$ problem with the same open boundary, on the other hand, has a much more complicated expression (also contains singularity). In other words, the methodology to be used in reducing complexities for $1 \mathrm{~d} / 2 \mathrm{~d} / 3 \mathrm{~d}$ problems and yet maintaining good accuracy also follows a very different process. We therefore deem it necessary to focus this paper only on a $1 \mathrm{~d}$ random medium problem. A higher dimension random medium problem requires another publication.

\section{Single Realization 1d Weak Scattering Random Media}

Let the $1 \mathrm{~d}$ random medium propagate along the $z$-direction. The total field (1) now simplifies to

$$
\begin{aligned}
& \epsilon_{m}(z) \partial_{t} E_{x, T_{m}}(z, t)+\sigma_{m}(z) E_{x, T_{m}}(z, t) \\
& \quad=-\partial_{z} H_{y, T_{m}}(z, t) \\
& \mu_{o} \partial_{t} H_{y, T_{m}}(z, t)=-\partial_{z} E_{x, T_{m}}(z, t)-f\left(z_{s}, t\right) .
\end{aligned}
$$

The background incident wave (2) also reduces to

$$
\begin{aligned}
& \epsilon_{B}(z) \partial_{t} E_{x, \text { inc }}(z, t)+\sigma_{B}(z) E_{x, \text { inc }}(z, t) \\
& \quad=-\partial_{z} H_{y, \text { inc }}(z, t) \\
& \mu_{o} \partial_{t} H_{y, \text { inc }}(z, t) \\
& \quad=-\partial_{z} E_{x, \text { inc }}(z, t)-f\left(z_{s}, t\right) .
\end{aligned}
$$

Depending on the distribution of $\epsilon_{B}(z)$ and $\sigma_{B}(z)$, this background incident wave $E_{x \text {,inc }}(z, t)$ excited with a single point $f\left(z_{s}, t\right)$ can be obtained in many different ways. For example, if it is a layered medium then the frequency domain boundary matching technique can give an exact solution, simply by taking the inverse transform of the frequency domain solution. For other more complicated profiles, it is necessary to evaluate this incident wave numerically. Lastly, the scattered field (3) written in terms of the background incident electric $E_{x, \text { inc }}(z, t)$ becomes

$$
\begin{aligned}
& \epsilon_{m}(z) \partial_{t} E_{x, S_{m}}(z, t)+\sigma_{m}(z) E_{x, S_{m}}(z, t) \\
& \quad=-\partial_{z} H_{y, S_{m}}(z, t)-\Delta \epsilon_{m}(z) \partial_{t} E_{x, \text { inc }}(z, t) \\
& \quad-\Delta \sigma_{m}(z) E_{x, \text { inc }}(z, t) \\
& \mu_{o} \partial_{t} H_{y, S_{m}}(z, t)=-\partial_{z} E_{x, S_{m}}(z, t) .
\end{aligned}
$$


Equivalently, the (17a) can also be written in terms of a mix of electric and magnetic incident fields:

$$
\begin{aligned}
\epsilon_{m}(z) \partial_{t} E_{x, S_{m}}(z, t)+\sigma_{m}(z) E_{x, S_{m}}(z, t) \\
=-\partial_{z} H_{y, S_{m}}(z, t)+\frac{\Delta \epsilon_{m}(z)}{\epsilon_{B}(z)} \partial_{z} H_{y, \text { inc }}(z, t) \\
\quad+\left[\frac{\Delta \epsilon_{m}(z)}{\epsilon_{B}(z)} \sigma_{B}(z)-\Delta \sigma_{m}(z)\right] E_{x, \text { inc }}(z, t) .
\end{aligned}
$$

A multiple-realization Monte-Carlo scheme obtains its scattered field ensemble average by either running (15) and then subtracting out the incident wave, or running (17) directly. In either case, the fluctuations $\epsilon_{m}(z)$ and $\sigma_{m}(z)$ in each realization are drawn (simulated) from a pre-defined set of statistics. These statistics in principle can come from a correlation function for which the solution to the governing S-PDE exists. Such an existence is a subject of Ito's Calculus [8], which will not be discussed here. For the purpose of demonstrating the accuracy of the proposed algorithm, we shall only use an exponential correction function by varying its spatial correction length $l_{c}$. To this end, an exponential correlation function is a convolution relation for a function $g(z)$ with its neighboring $g(z+\tau)$ satisfying $R(\tau)=\langle g(z) g(z+\tau)\rangle=g_{r m s}^{2} e^{-|\tau| / l_{c}}$ where the root-mean-square is evaluated at $R(0)=g_{r m s}^{2}$. The correlation length $l_{c}$ is therefore a scale measuring how well two different points along the function $g(z)$ and $g(z+\tau)$ are correlated. A digital filter technique for realizing this correlation function was provided in [1]. Numerical examples to be discussed in a later section actually use a slightly different implementation provided by Ilker Capoglu in our lab (this code is also available upon request).

It is also important to point out that one should not mistakenly conclude that biological cells follow such a simple correlation function. In fact, characterizing experimental correlation functions as a clinical marker for distinguishing a benign from a cancerous cell is an ongoing work in our lab [18]-[20] which motivated the need for a single-realization random medium Monte-Carlo scheme. For example, large sets of scattered field data collected from different types of cells supplied by a hospital are currently being processed for the purpose of modeling the statistical random medium problem. The verification of the experimental data against possible models therefore requires stochastic Maxwell's partial differential equations for which without a single-realization scheme would be an extremely inefficient task to process.

Next, in a random medium with a weak scattering problem the background is often composed of homogeneous medium having constant $\epsilon_{B}(z)$ and $\sigma_{B}(z)$ everywhere. Scattering by dust particles is such an example. Similarly, the actual variations of $\epsilon_{m}(z)$ observed in biological cells also appear to be small enough such that identifying a constant $\epsilon_{B}$ to form $\epsilon_{m}(z)=$ $\epsilon_{B}+\Delta \epsilon_{m}(z)$ satisfies the weak scattered field constraint, i.e, $\epsilon_{B}$ is much larger than $\Delta \epsilon_{m}(z)$. In fact, $\sigma_{m}(z)$ inside the cell is also ignored (measuring its actual value is another practical challenge) since at the optical frequency the displacement current $\epsilon_{m} \partial_{t} E_{x, S_{m}}$ is believed to be significantly larger than the diffusion current $\sigma_{m} E_{x, S_{m}}$. Upon employing the linearly inde- pendent partition (8) together with the lossless and uniform assumptions, the complete set of the $1 \mathrm{~d}$ iterative scattered field PDE now reduces

$$
\begin{aligned}
& \epsilon_{B} \partial_{t} E_{x, S_{m}}^{(q+1)}(z, t)=-\partial_{z} H_{y, S_{m}}^{(q+1)}(z, t)-J^{(q)}\left(z_{k}, t\right) \\
& \mu_{o} \partial_{t} H_{y, S_{m}}^{(q+1)}(z, t)=-\partial_{z} E_{x, S_{m}}^{(q+1)}(z, t) .
\end{aligned}
$$

The polarization current density $J^{(q)}\left(z_{k}, t\right)$, which is an independent source to the PDE and in the anticipation for the discrete implementation to be fully discussed next, will be expressed in terms of the unit sample $\delta\left(k^{\prime}-k\right)$ having non-zero value only at $k^{\prime}=k$

$$
J_{\epsilon, m}^{(q)}\left(z_{k}, t\right)=\sum_{k^{\prime}=0}^{N-1} \Delta \epsilon_{m}\left(z_{k}\right) \delta\left(k-k^{\prime}\right) \partial_{t} E_{x, S_{m}}^{(q)}\left(z_{k}, t\right)
$$

where $z_{k}=k \Delta z$, and both $k$ and $k^{\prime}$ are integers.

The methodology tailored to the FDTD scheme for an arbitrary order $q$ accuracy is now developed. In a discrete formulation the incident wave exists only at discrete points $E_{x, \text { inc }}(z, t)=E_{x, \text { inc }}\left(z_{k}, t\right)$ where $\Delta z$ assumes the FDTD discrete spacing. Similarly, the random medium statistics are also sampled at $\Delta \epsilon_{m}(z)=\Delta \epsilon_{m}\left(z_{k}\right)$, which will be denoted by $\Delta \epsilon_{m, k}$. Let the random medium start at $k=0$ and extend to a finite length of $N-1$ points, i.e., $\Delta \epsilon_{m, k}=0$ exterior to this region. This discrete sampling process is depicted in Fig. 1 with part (a) showing the temporal derivative of the incident field at the discrete points, (b) sampling $\Delta \epsilon_{m, k}$ with constant value within the discrete spacing $\Delta z$, and (c) the same $\Delta z$ to be used in the FDTD discrete spacing. The independent boundary value or excitation $f\left(z_{s}\right)$ responsible for generating the incident wave is assigned at a distance exterior (to the left) to the random medium. It is also important to point out that spacing $\Delta z$ needs to be small enough so that statistical details in the fluctuations $\Delta \epsilon_{m}(z)$ along the $z$-direction are properly characterized. In other words, the discretization spacing $\Delta z$ required in a random medium problem in general is dictated by the statistical spatial correlation length, which in this case is the parameter $l_{c}$, rather than the more conventional criterion of wavelength per sample point.

1) First Order Iteration: The above discrete diagram makes it clear that in a discrete formulation the polarization current has a collection of $N$ discrete point sources distributing along the discrete $z_{k}$. This means that the first order iteration PDE (19) at $q=0$ becomes

$$
\begin{aligned}
& \epsilon_{B} \partial_{t} E_{x, S_{m}}^{(1)}(z, t)=-\partial_{z} H_{y, S_{m}}^{(1)}(z, t)-J_{\epsilon, m}^{(0)}\left(z_{k}, t\right) \\
& \mu_{o} \partial_{t} H_{y, S_{m}}^{(1)}(z, t)=-\partial_{z} E_{x, S_{m}}^{(1)}(z, t)
\end{aligned}
$$

with the point polarization current densities composing of the incident temporal derivative scaled by the statistical

$$
J_{\epsilon, m}^{(0)}\left(z_{k}, t\right)=\sum_{k^{\prime}=0}^{N-1} \Delta \epsilon_{m, k} \delta\left(k-k^{\prime}\right) \partial_{t} E_{x, \text { inc }}\left(z_{k}, t\right) .
$$

Note that the excitation density $J_{\epsilon, m}^{(0)}\left(z_{k}, t\right)$ needs to include all $0 \leq k \leq N-1$, which exactly has $N$ points. To this 


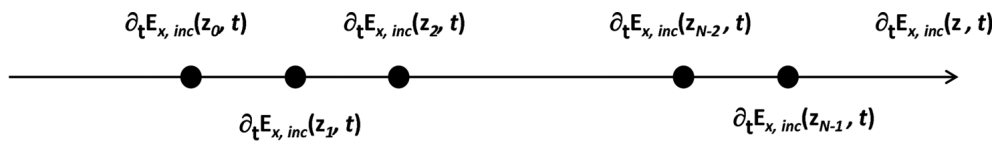

(a)

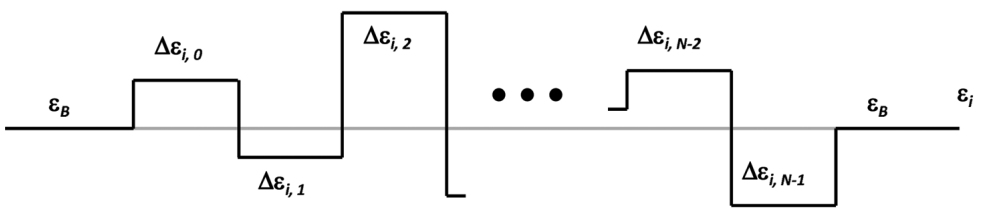

(b)

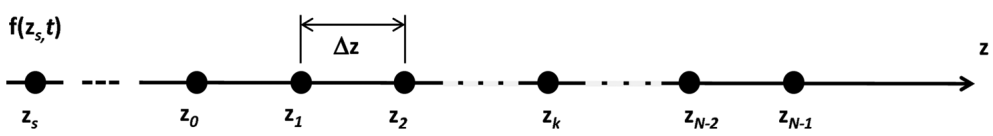

(c)

Fig. 1. Discrete $1 \mathrm{~d}$ random medium with $N$ equal spacing samplings (a) temporal derivative incident wave at every $z_{k}$ (b) sampled dielectric values constant within each $\Delta z$ (c) arrangement of FDTD discrete spacing $\Delta z$ which must be small enough to account for statistical variations along the $z$-axis.

end, if only the first order accuracy is required then the singlerealization ensemble average formulation simply comes from the expectation value of this equation, and the process ends here. Specifically,

$$
\begin{aligned}
& \epsilon_{B} \partial_{t}\left\langle E_{x, S}^{(1)}(z, t)\right\rangle=-\partial_{z}\left\langle H_{y, S}^{(1)}(z, t)\right\rangle-\left\langle J_{\epsilon}^{(0)}\left(z_{k}, t\right)\right\rangle \\
& \mu_{o} \partial_{t}\left\langle H_{y, S}^{(1)}(z, t)\right\rangle=-\partial_{z}\left\langle E_{x, S}^{(1)}(z, t)\right\rangle
\end{aligned}
$$

where

$$
\left\langle J_{\epsilon}^{(0)}\left(z_{k}, t\right)\right\rangle=\sum_{k^{\prime}=0}^{N-1}\left\langle\Delta \epsilon_{k}\right\rangle \delta\left(k-k^{\prime}\right) \partial_{t} E_{x, \text { inc }}\left(z_{k}, t\right) .
$$

Being an ensemble average formulation, notice that none of the variables involves the realization $m$. Also, the solution to this single-realization equation can now be obtained in many different ways. In particular, the complexity associated with generating the polarization currents will be exactly $\mathrm{N}$ points. Keep in mind that every incident $E_{x, \text { inc }}\left(z_{k}, t\right)$ is a known deterministic variable. Lastly, this ensemble polarization if replaced with the incident magnetic field becomes $-\sum_{k^{\prime}=0}^{N-1}\left\langle\Delta \epsilon_{k}\right\rangle \delta(k-$ $\left.k^{\prime}\right) \tilde{\partial}_{z} H_{y, \text { inc }}\left(z_{k}, t\right) / \epsilon_{B}\left(z_{k}\right)$, where $\tilde{\partial}_{z}$ signifies a discrete counterpart of $\partial_{z}$.

2) Second Order Iteration: Similarly, the second order iteration $q=1$ is evaluated by

$$
\begin{aligned}
& \epsilon_{B} \partial_{t} E_{x, S_{m}}^{(2)}(z, t)=-\partial_{z} H_{y, S_{m}}^{(2)}(z, t)-J_{\epsilon, m}^{(1)}\left(z_{k}, t\right) \\
& \mu_{o} \partial_{t} H_{y, S_{m}}^{(2)}(z, t)=-\partial_{z} E_{x, S_{m}}^{(2)}(z, t)
\end{aligned}
$$

with the discrete polarization current densities coming from

$$
J_{\epsilon, m}^{(1)}\left(z_{k}, t\right)=\sum_{k^{\prime}=0}^{N-1} \Delta \epsilon_{m, k} \delta\left(k-k^{\prime}\right) \partial_{t} E_{x, S_{m}}^{(1)}\left(z_{k}, t\right) .
$$

Again, this current density also needs to sample all $N$ points. As stated earlier, obtaining a second order iteration single-realization scheme requires the polarization current to be fully expressed in terms of the deterministic incident wave and all other ensemble parameters. This can be facilitated by the fact that the first order scattered fields, because of $N$ discrete independent excitations, must have a functional relation

$$
E_{x, S_{m}}^{(1)}\left(z_{k}, t\right)=-\gamma \sum_{k^{\prime}=0}^{N-1} \Delta \epsilon_{m, k^{\prime}} \partial_{t} E_{x, \text { inc }}\left(z_{k^{\prime}}, t+D_{k, k^{\prime}}^{(1)}\right)
$$

where the unit consistency scaling for an FDTD implementation should be set as $\gamma=\Delta t / \epsilon_{B}$ and the shift $D_{k, k^{\prime}}^{(1)}$ accounts for the temporal delay which in general depends on the distance between $k^{\prime}$ and $k$. Numerical efficient for a random medium problem therefore depends strongly on how this delay relation is implemented.

To begin, an $N^{2}$ complexity associated with the excitations is clear, substituting (27) into (26) results in one summation calling another summation. Avoiding this power factor complexity follows from the following observations. First, the incident wave in this case is coming from the left with $z_{s}$ exterior to the inhomogeneity. Next, a plane wave has a property $E_{x, \text { inc }}(z \pm k \Delta z, t)=E_{x, \text { inc }}(z, t \mp k \Delta t)$. Furthermore, the scattered field $E_{x, S_{m}}^{(1)}(z, t)$ can be decomposed into both left and right propagating waves, because every point source generates a wave going both directions. In particular, a right propagation wave will be in phase with $E_{x, \text { inc }}$ whereas the left propagation accumulates double delays (passing $z_{k}$ first and then bouncing back). For example, at the furthest point $z_{k=N-1}$ there is a superposition of in phase propagating $\sum_{k^{\prime}=0}^{N-1} \epsilon_{m, k^{\prime}} \partial_{t} E_{x, \text { inc }}\left(z_{N-1}, t\right)$ wave which when scaled by $\epsilon_{m, N-1}$ and after taking the first temporal derivative forms the polarization current density for the second order iteration that accounts for the internal reflections within the random medium. On the other hand, at $z_{k=0}$ the left scattered wave responsible for the internal reflections within the inhomogeneities comes from the superposition of $\sum_{k^{\prime}=1}^{N-1} \epsilon_{m, k^{\prime}} \partial_{t} E_{x, \text { inc }}\left(z_{0}, t-2 k^{\prime} \Delta t\right)$. These properties all together allow the polarizations to be separated into the right and left current densities

$$
J_{\epsilon, m}^{(1)}\left(z_{k}, t\right)=J_{R, m}^{(1)}\left(z_{k}, t\right)+J_{L, m}^{(1)}\left(z_{k}, t\right)
$$


where

$$
\begin{aligned}
J_{R, m}^{(1)}\left(z_{k}, t\right)= & -\gamma \sum_{k^{\prime \prime}=0 k^{\prime}=0}^{N-1} \sum_{k^{\prime \prime}} \Delta \epsilon_{m, k} \Delta \epsilon_{m, k^{\prime}} \delta\left(k-k^{\prime \prime}\right) \\
& \times \partial_{t}^{2} E_{x, \text { inc }}\left(z_{k}, t\right) \\
J_{L, m}^{(1)}\left(z_{k}, t\right)= & -\gamma \sum_{k^{\prime \prime}=0}^{N-2} \sum_{k^{\prime}=k^{\prime \prime}+1}^{N-1} \Delta \epsilon_{m, k} \Delta \epsilon_{m, k^{\prime}} \delta\left(k-k^{\prime \prime}\right) \\
& \times \partial_{t}^{2} E_{x, \text { inc }}\left(z_{k}, t-2\left(k^{\prime}-k^{\prime \prime}\right) \Delta t\right)
\end{aligned}
$$

Equivalently, the left or the out of phase propagating polarization can also be written as

$$
\begin{aligned}
J_{L, m}^{(1)}\left(z_{k}, t\right)= & -\gamma \sum_{k^{\prime \prime}=0}^{N-2} \sum_{k^{\prime}=k^{\prime \prime}+1}^{N-1} \Delta \epsilon_{m, k} \Delta \epsilon_{m, k^{\prime}} \delta\left(k-k^{\prime \prime}\right) \\
& \times \partial_{t}^{2} E_{x, \text { inc }}\left(z_{k}+2\left(k^{\prime}-k^{\prime \prime}\right) \Delta z, t\right) .
\end{aligned}
$$

The advantage of this separation is that the in phase source excitation $J_{R . m}^{(1)}$ reduces to an $N$ complexity, because $\partial_{t}^{2} E_{x, \text { inc }}\left(z_{k}, t\right)$ depends only on $k$. However, the source complexity for the out of phase term $J_{L, m}^{(1)}$ is $(N-1) N / 2$, because its incident excitations depend also on $k^{\prime}$ and $k^{\prime \prime}$. Depending on the applications, this quadratic complexity may still need to be reduced further. There are ways to accomplish this task. This will be systematically addressed after the completion of the single-realization ensemble average formulation.

To this end, a second order accuracy multiple-realization Monte-Carlo scheme simply comes from the sum of the two approximated solutions $E_{x, S_{m}} \approx E_{x, S_{m}}^{(1)}+E_{x, S_{m}}^{(2)}$ and $H_{x, S_{m}} \approx H_{x, S_{m}}^{(1)}+H_{x, S_{m}}^{(2)}$. Taking their expectation values therefore completes the ensemble average readily to be evaluated with a single-realization scheme. Specifically, the sum of (23) and the ensemble of (25) which after substituting their corresponding polarization current densities reduces to

$$
\begin{aligned}
& \epsilon_{B} \partial_{t}\left\langle E_{x, S}(z, t)\right\rangle=-\partial_{z}\left\langle H_{y, S}(z, t)\right\rangle \\
& \quad-\sum_{k^{\prime}=0}^{N-1}\left\langle\Delta \epsilon_{m, k}\right\rangle \delta\left(k-k^{\prime}\right) \partial_{t} E_{x, \text { inc }}\left(z_{k}, t\right) \\
& \quad+\gamma \sum_{k^{\prime \prime}=0 k^{\prime}=0}^{N-1} \sum_{k^{\prime \prime}}\left\langle\Delta \epsilon_{k} \Delta \epsilon_{k^{\prime}}\right\rangle \delta\left(k-k^{\prime \prime}\right) \partial_{t}^{2} E_{x, \text { inc }}\left(z_{k}, t\right) \\
& \quad+\gamma \sum_{k^{\prime \prime}=0}^{N-2} \sum_{k^{\prime}=k^{\prime \prime}+1}^{N-1}\left\langle\Delta \epsilon_{k} \Delta \epsilon_{k^{\prime}}\right\rangle \delta\left(k-k^{\prime \prime}\right) \\
& \quad \times \partial_{t}^{2} E_{x, \text { inc }}\left(z_{k+2\left[k^{\prime}-k^{\prime \prime}\right]}, t\right) \\
& \mu_{o} \partial_{t}\left\langle H_{y, S}(z, t)\right\rangle=-\partial_{z}\left\langle E_{x, S}(z, t)\right\rangle .
\end{aligned}
$$

Here we have made use of the temporal delay (30) equivalence. Unlike the first order iteration (23), this second order accuracy single-realization includes the second order cross-correlation $\Delta \epsilon_{k^{\prime}}$ and $\Delta \epsilon_{k}$ statistics which account for the internal reflections within the random medium. It therefore is capable of dealing with a zero mean $\left\langle\Delta \epsilon_{k}\right\rangle=0$. Again, the solution to this single-realization scheme can be evaluated with any standard mathematical techniques. However, we will continue with the standard Yee's 1d algorithm. Because an expectation is simply a single value, (31) fundamentally amounts to a standard pure scattered field formulation. Details of how to translate a pure scattered field formulation into FDTD implementation can be found in [12], [21], hence they will not be repeated here.

For completeness, we add that if lossy random medium problem is of interest then additional ensemble average terms such as the background $\sigma_{B}(z)\left\langle E_{x, S}(z, t)\right\rangle$, the first order polarizations $\sum_{k^{\prime}=0}^{N-1}\left\langle\Delta \sigma_{k^{\prime}}\right\rangle \delta\left(k-k^{\prime}\right) E_{x, \text { inc }}\left(z_{k}, t\right)$, and the second order polarizations $\sum_{k^{\prime \prime}=0}^{N-1} \sum_{k^{\prime}=0}^{N-1}\left\langle\Delta \sigma_{k} \Delta \sigma_{k^{\prime}}\right\rangle \delta(k-$ $\left.k^{\prime \prime}\right) \partial_{t} E_{x, \text { inc }}\left(z_{k^{\prime \prime}}, t+D_{k^{\prime}, k}^{(1)}\right)$ will need to be added in the above lossless formulation (31). In this case, the lossy background incident wave $E_{x \text {.inc }}$ also needs to be evaluated from (16) with the corresponding non-zero $\sigma_{B}$. Lastly, the technique developed here can be adopted to address background consisting of layered mediums. In particular, this incident could be obtained more efficiently using the frequency domain formulation, because of its analytic solution.

3) Polarization Complexity Reduction: We now show that there are ways to implement $\left\langle J_{L}^{(1)}\left(z_{k}, t\right)\right\rangle$ more efficiently. The key idea is to make use of the Fourier transform linear phase shift and constant time delay relationship. For example, taking its Fourier transform and the ensemble average of (29b), and defining the linear phase $a=e^{-j \omega \Delta t}$ result in

$$
\begin{aligned}
\left\langle\hat{J}_{L}^{(1)}\left(z_{k}, \omega\right)\right\rangle=\gamma \sum_{k^{\prime \prime}=0}^{N-2} \sum_{k^{\prime}=k^{\prime \prime}+1}^{N-1}\left\langle\Delta \epsilon_{k} \Delta \epsilon_{k^{\prime}}\right\rangle a^{2\left(k^{\prime}-k^{\prime \prime}\right)} \\
\times \delta\left(k-k^{\prime \prime}\right) \omega^{2} \hat{E}_{x, \text { inc }}\left(z_{k}, \omega\right) .
\end{aligned}
$$

where $\hat{E}_{x, \text { inc }}\left(z_{k}, \omega\right)$ is the transform pair of $E_{x, \text { inc }}\left(z_{k}, t\right)$. Similar to the in phase incident (29a), the polarization current density in this transform domain now also has an $N$ complexity. Again, the incident wave in this case also depends only on $k$. In fact, to avoid evaluating at each $z_{k}$ this incident wave should be replaced with $\hat{E}_{x, \text { inc }}\left(z_{k}, \omega\right)=a^{k} \hat{E}_{x, \text { inc }}\left(z_{0}, \omega\right)$. Following the technique successfully employed in the AFP or O-AFP [22], [23], the time sequences are recovered from the inverse transform using FFT (fast Fourier transform). It should be said that if all the temporal data are stored away before the run-time, then for larger $N$ a potential disadvantage in this implementation would come from expensive memory requirement. This memory storage can be avoided if the transformation is performed over the space. For example, recall also [22], [23] that space and time in a plane wave has a simple wave relation $\hat{E}_{x, \text { inc }}\left(z_{k}, \omega\right) e^{j[\kappa \Delta z k-\omega \Delta t s]}$ where $\kappa$ is the wavenumber and $s$ refers to the discrete time-step $t_{s}=s \Delta t$, and hence $\omega=$ $c \kappa$ with the phase velocity $c=\Delta z / \Delta t$. This means that the linear phase can be replaced with $a=e^{-j \omega \Delta t}=e^{-j \kappa \Delta z}$, and $\hat{E}_{x, \text { inc }}\left(z_{k}, \omega\right)$ can also be converted to $\hat{E}_{x \text {,inc }}\left(\kappa, t_{s}\right)$. In this way the FFT can be evaluated at every time-step $t_{s}$.

Another possibility is to introduce further approximations. For example, consider the first $z_{k=0}$ which also has the longest summation term in (32)

$$
\begin{aligned}
{\left[\left\langle\Delta \epsilon_{0} \Delta \epsilon_{1}\right\rangle a^{2}+\left\langle\Delta \epsilon_{0} \Delta \epsilon_{2}\right\rangle a^{4}+\cdots\right.} & \left.+\left\langle\Delta \epsilon_{0} \Delta \epsilon_{N-1}\right\rangle a^{2(N-1)}\right] \\
& \times \omega^{2} \hat{E}_{x, \text { inc }}\left(z_{0}, \omega\right) .
\end{aligned}
$$


In one extreme this excitation can be approximated with a single source

$$
\tilde{A}_{0} e^{-j \omega \tilde{d}_{0} \Delta t} \omega^{2} \hat{E}_{x, \text { inc }}\left(z_{0}, \omega\right) \text {. }
$$

where the effective magnitude $\tilde{A}_{0}$ and delay $\tilde{d}_{0}$ can be systematically obtained from a curve-fitting procedure. Its corresponding time-domain translates to

$$
-\tilde{A}_{0} \partial_{t}^{2} E_{x, \text { inc }}\left(z_{0}, t-\tilde{d}_{0}\right) .
$$

If every second order polarization current density is approximated with such a single source replacement then the complexity associated with the source will again become linear. For an exponential correlation function having a shorter correlation length $l_{c}$ our numerical experiments had indeed verified that such an approximation works very well. This is because points that are farther apart, because they are not well-correlated, tend to add incoherently. Obviously, adding more terms in the approximation helps improving the accuracy. Note also that the last term $\left\langle\Delta \epsilon_{N-2} \Delta \epsilon_{N-1}\right\rangle a^{2} \omega^{2} \hat{E}_{x, \text { inc }}\left(z_{N-2}, \omega\right)$ needs no approximation.

\section{NumERICAL EXAMPLES}

All simulations assume $N=3500$ points within which the random medium statistics fluctuates. For an FDTD spacing $\Delta z=3 \mathrm{~nm}$ these points translate to a cell having $10.5 \mu \mathrm{m}$ thickness. The incident wave excitation is a modulated Gaussian with a bandwidth expanding approximately $500 \mathrm{~nm}$ to 700 $\mathrm{nm}$ and centering at $\lambda_{0}=600 \mathrm{~nm}$. To eliminate numerical dispersion mismatch [24], [25], both the multiple-realization scheme (17) and single-realization scheme (31) also use the same stability value $S=0.95$. This setup translates to approximately 200 sample points per wavelength. The uniform background for all examples also assumes an optical index reflection $n=n_{o}+\Delta n$ with $n_{o}=1.45$ and fluctuating at a maximum of $\Delta n= \pm 0.1$ and its relation to relative permittivity is $\epsilon_{r}=\sqrt{n}$. For illustration purposes, some large discrete points are intentionally added to both sides of the sample to make room for displaying or recording the forward and backward scattered fields (no absorbing boundary condition). In the discrete spatial display, the random medium actually resides within $3500 \Delta z \leq z_{k} \leq 7000 \Delta z$, and all other points exterior this region is the uniform background (hence $k$ starts at 3500, rather than 0 , and ends at 7000). Lastly, since (32) fundamentally amounts to introducing additional data structure to the iteration formulation, all demonstrations use the direct implementation (31) without any further approximation.

Figs. 2 and 3 show simulation results for an exponential correlation function with correlation length of $l_{c}=4 \Delta z=0.02 \lambda_{o}$. Fig. 2(a) plots a sample of this correlation function over space $z_{k}$. Because of this small correlation length, the index reflection $n\left(z_{k}\right)$ fluctuates very quickly, i.e., points that are larger than $l_{c}$ distance away practically fluctuate independently. Fig. 2(b) shows the actual ensemble scattered field $\left\langle E_{x, S}\left(z_{k}, t\right)\right\rangle$ averaged over $M=4000$ realizations, plotting over space $z_{k}$ at one instant $t_{s}$. The dashed blue line is simulated with the multiple-realization Monte-Carlo whereas the dotted red line comes from the proposed single-realization scheme. The two

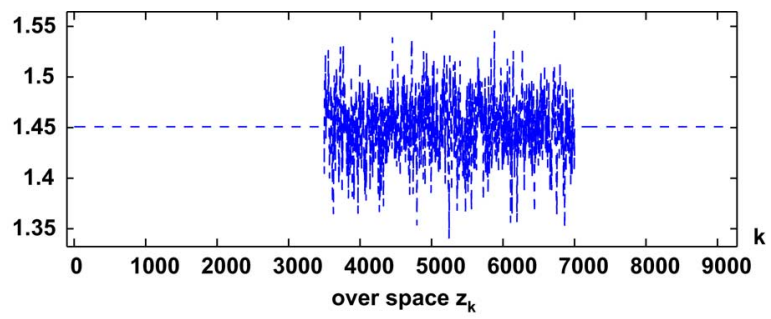

(a)

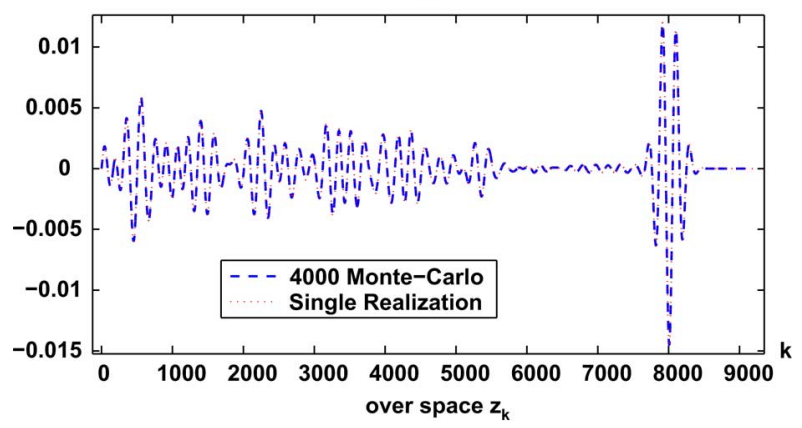

(b)

Fig. 2. Exponential correlation function simulation results with correlation $l_{c}=4 \Delta z=0.02 \lambda_{o}$ (a) a sample of a statistical optical index $n\left(z_{k}\right)$ fluctuating around a mean of 1.45 , and (b) ensemble scattered field $\left\langle E_{x, S}(z, t)\right\rangle$ along $z_{k}$ at some time $t_{s}$ for both the multiple-realization and proposed single-realization schemes averaging over 4000 realizations. (a) Index Reflection $n\left(z_{k}\right)$ at $l_{c}=4 \Delta z$; (b) Ensemble Scattered Field $\left\langle E_{x, S}\left(z_{k}, t_{n},\right)\right\rangle$.

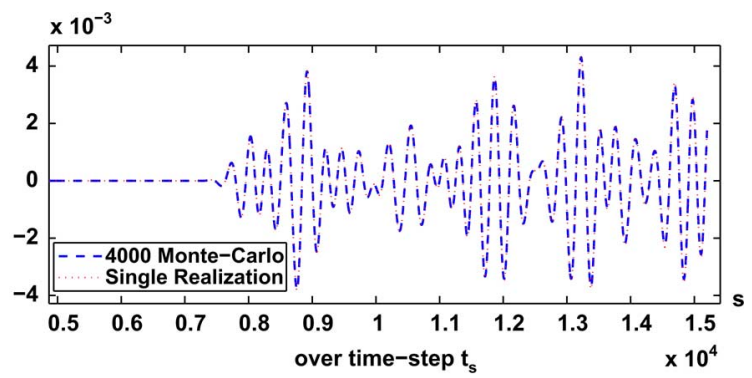

(a)

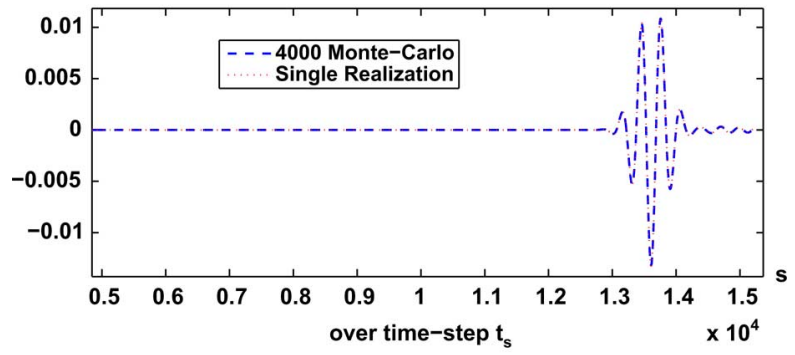

(b)

Fig. 3. Scattered fields of Fig. 2 observed over time (a) back scattering recorded at $z_{b}$ a few points before entering the random medium (b) forward (transmitted) scattering at $z_{f}$ a few points after leaving the random medium. (a) Ensemble Back Scattered Field $\left\langle E_{x, S}\left(z_{k}, t_{n},\right)\right\rangle$; (b) Ensemble Forward Scattered Field $\left\langle E_{x, S}\left(z_{k}, t_{n},\right)\right\rangle$.

techniques clearly show an excellent agreement. The same holds true over the temporal distribution. Specifically, Fig. 3(a) plots the back scattering field recorded at some fixed point $z_{b}$ before entering the random medium and Fig. 3(b) plots the forward (transmitted) scattering field collected at $z_{f}$ a few points after leaving the random medium. It should be pointed 


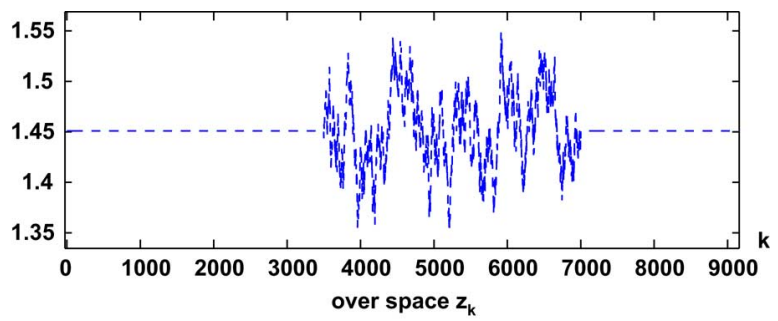

(a)

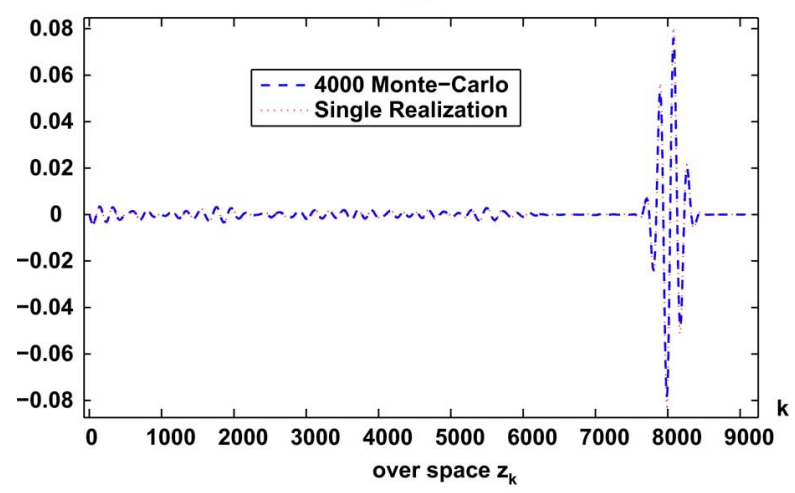

(b)

Fig. 4. Exponential correlation function simulation results with correlation $l_{c}=85 \Delta z=0.245 \lambda_{o}$ (a) a sample of a statistical optical index $n\left(z_{k}\right)$ fluctuating around a mean of 1.45 , and (b) ensemble scattered field $\left\langle E_{x, S}(z, t)\right\rangle$ along $z$ at some time $t_{n}$ for both the multiple-realization and proposed single-realization schemes averaging over 4000 realizations. (a) Index Reflection $n\left(z_{k}\right)$ at $l_{c}=85 \Delta z ;\left(\right.$ b) Ensemble Scattered Field $\left\langle E_{x, S}\left(z_{k}, t_{n},\right)\right\rangle$.

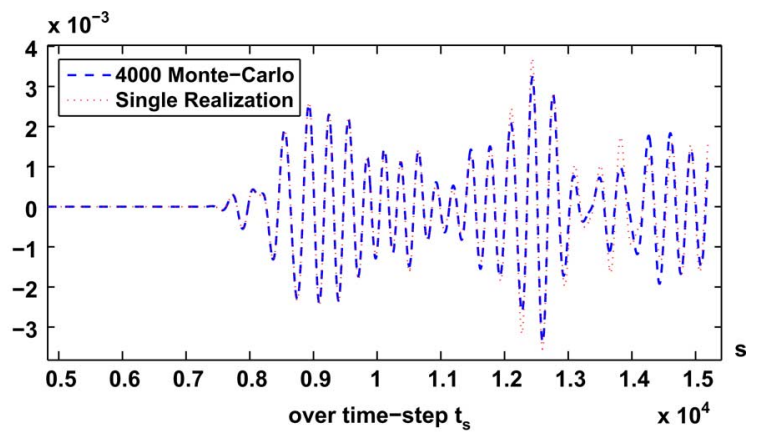

(a)

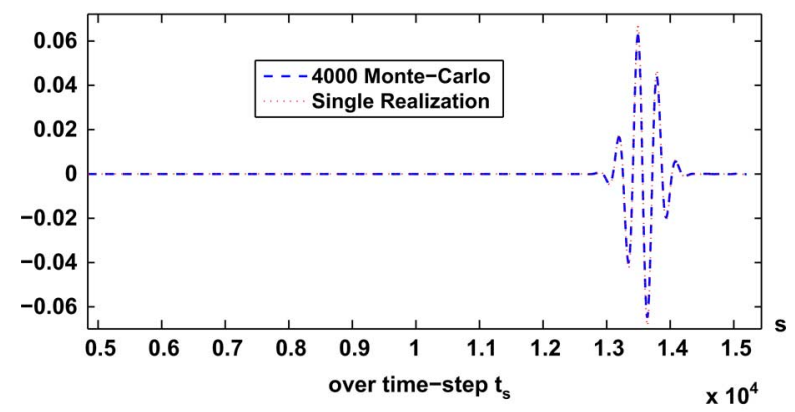

(b)

Fig. 5. Scattered fields of Fig. 4 observed over time (a) back scattering recorded at $z_{b}$ a few points before entering the random medium (b) forward (transmitted) scattering at $z_{f}$ a few points after leaving the random medium. (a) Ensemble Back Scattered Field $\left\langle E_{x, S}\left(z_{k}, t_{n},\right)\right\rangle$; (b) Ensemble Forward Scattered Field $\left\langle E_{x, S}\left(z_{k}, t_{n},\right)\right\rangle$.

that the accuracy of the proposed solution is valid for arbitrary realization $M$.

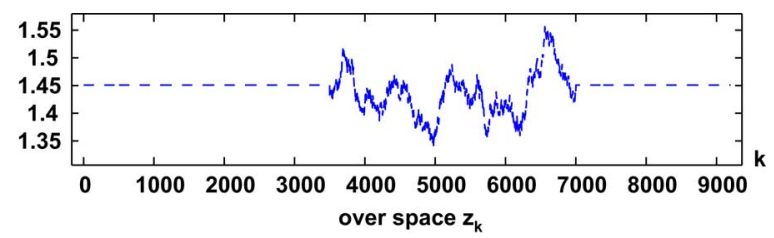

(a)

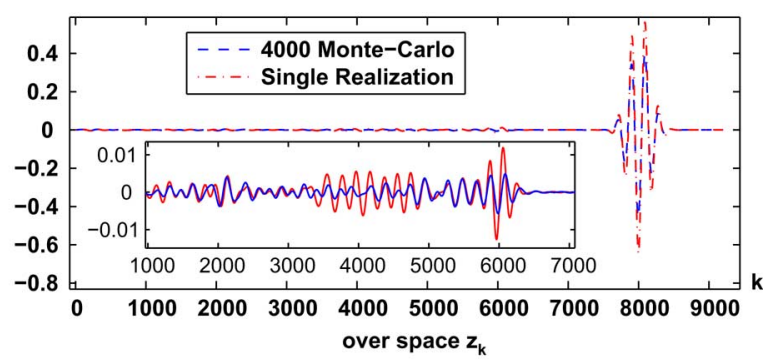

(b)

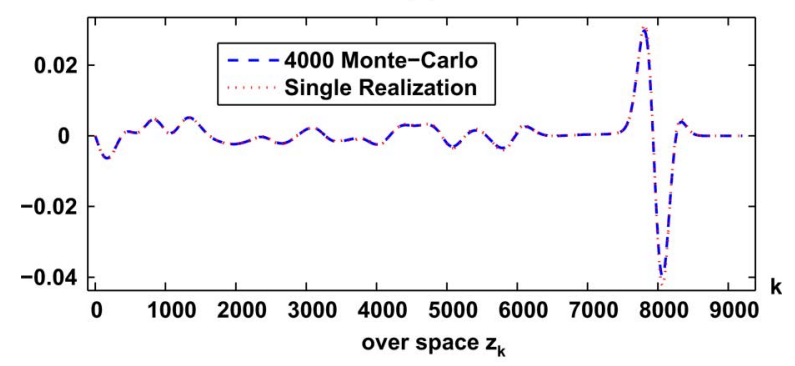

(c)

Fig. 6. Exponential correlation function simulation results with correlation $l_{c}=250 \Delta z=1.25 \lambda_{o}$ (a) a sample of a statistical optical index $n\left(z_{k}\right)$ fluctuating around a mean of 1.45 (b) ensemble scattered field $\left\langle E_{x, S}(z, t)\right\rangle$ along $z$ at some time $t_{n}$ for both the multiple-realization and proposed single-realization schemes averaging over 4000 realizations, and (c) the same simulation but with incident wave not modulating with the sine wave. (a) Index Reflection $n\left(z_{k}\right)$ at $l_{c}=250 \Delta z$; (b) Ensemble Scattered Field $\left\langle E_{x, S}\left(z_{k}, t_{n},\right)\right\rangle$ (c) Ensemble Scattered Field $\left\langle E_{x, S}\left(z_{k}, t_{n},\right)\right\rangle$.

Figs. 4 and 5 show simulation results for $l_{c}=85 \Delta z=$ $0.425 \lambda_{o}$ but keeping all other parameters unchanged. In comparison with the shorter $l_{c}$ above, the optical index $n\left(z_{k}\right)$ in Fig. 4(a) now fluctuates much slower. However, the scattered fields as shown in the spatial $4 \mathrm{~b}$ and temporal $5 \mathrm{a}, \mathrm{b}$ are still in a relatively agreement. For completeness, we would like to add that it is at this large correlation $l_{c}$ that the single source replacement (33) or (35) starts becoming ineffective.

Lastly, Fig. 6 demonstrates a large correlation $l_{c}=250 \Delta z=$ $1.25 \lambda_{o}$ for which the second order iteration will also become ineffective. Again, this very well correlated $n\left(z_{k}\right)$ having all other parameters unchanged otherwise is plotted in Fig. 6(a) with its ensemble scattered field over space showing on part $b$. Specifically, the inset showing in Fig. 6(b) demonstrates that the two simulation results no longer follow each other. This can be understood also through the expansion (33) where each of the coefficients now will add coherently. This means that their corresponding $\left|E_{x, S_{m}}^{(0)}\right|$ much smaller than $\left|E_{x, S_{m}}^{(1)}\right|$ is no longer valid. As a result, the assumption $E_{x, S_{m}} \approx E_{x, S_{m}}^{(0)}+E_{x, S_{m}}^{(1)}$ no longer forms a good approximation and hence additional iteration $E_{x, S_{m}}^{(q)}$ becomes necessary. However, as stated earlier, the accuracy of the proposed iterative formulation in general depends also on the incident wave spectrum. This is demonstrated 
in Fig. 6(c) where in this case the incident is not modulated with the sine wive. Notice that the two simulations again follow each other.

\section{DISCUSSION AND CONCLUSION}

Our numerical examples demonstrate that the proposed single-realization works very well for a weak scattering random medium problem characterized by a smaller $\Delta n$, $l_{c}$, and incident wave having a lower spectral content. It is therefore an effective algorithm for evaluating biological cells believed to fluctuate at a shorter correlation length. It loses its effectiveness when the correlation length $l_{c}$ is commensurable to the wavelength of the incident wave. This is a consequence of the fact that for a shorter $l_{c}$ the second-order statistics or the cross-correlation $\Delta \epsilon_{k, m} \epsilon_{k^{\prime}, m}$ statistically will often add more incoherently (more cancellations). For example, the polarization $\sum_{k^{\prime}=0}^{k}\left\langle\Delta \epsilon_{k} \Delta \epsilon_{k^{\prime}}\right\rangle \partial_{t}^{2} E_{x, \text { inc }}\left(z_{k}, t\right)$ and its neighbor $\sum_{k^{\prime}=0}^{k+1}\left\langle\Delta \epsilon_{k+1} \Delta \epsilon_{k^{\prime}}\right\rangle \partial_{t}^{2} E_{x, \text { inc }}\left(z_{k+1}, t\right)$ for a shorter $l_{c}$ will tend to cancel each other. On the other hand, a longer $l_{c}$ will make these terms almost always add constructively. In the latter case, the magnitudes of $\left\langle E_{x, S}^{(1)}\right\rangle$ and $\left\langle E_{x, S}^{(0)}\right\rangle$ can become commensurable. Such a problem needs to be resolved with additional iterations $\sum_{k}^{N-1} \Delta \epsilon_{k, m} \partial_{t} E_{x, S_{m}}^{(q)}\left(z_{k}, t\right)$ with $q>1$. In particular, if the cross-correlation $\Delta \epsilon_{k, m} \epsilon_{k^{\prime}, m}$ is perfectly correlated over a long wavelength, i.e., $\Delta \epsilon_{k, m}$ and $\Delta \epsilon_{k^{\prime}, m}$ fluctuate exactly the same way over the entire $N$ points, then this iterative methodology may lose its effectiveness if $N$ occupies a few wavelength. In terms of Born's approximation insight [17], this is because there will be no internal multi-reflection cancellations within the medium to give rise to a weak scattering phenomenon.

This reasoning also helps understand the less accurate results reported in [3], [4]. For example, with some algebraic manipulations it is not difficult to show that their variance wave and the present first-order iteration single-realization scattered wave formulation (23) and (24) if included all the lossy terms will differ only by a scaling related to their proposed correlation coefficients $\rho_{\epsilon}$ and $\rho_{\sigma}$. This can be facilitated through (18), e.g., replace $\epsilon_{m}$ and $\sigma_{m}$ with $\epsilon_{B}$ and $\sigma_{B}$ and then take the expectation operation. On the other hand, each of their samples has a correlation length perfectly correlated over a few wavelengths (their sample are embedded in the layered mediums). As demonstrated in Fig. 6(a) and (b), a relatively high correlated statistics is still very difficult to be resolved even with the second order iteration formulation. Hence, a first order approximation should not expect to outperform its corresponding higher order counterpart.

Lastly, the principle idea that a weak scattering can be approximated with an iterative formulation also applies to $2 \mathrm{~d} / 3 \mathrm{~d}$ random mediums. As demonstrated, to improve accurate it is necessary to account for all necessary higher order internal reflections within the random medium. The challenge is therefore in the numerical efficiency. For example, further symmetry and reducing internal reflections which may not contribute constructively to the ensemble averages will need to be identified. If these redundancies are not employed then the formulation may not be an effective solution for an accuracy higher than a second-order iteration.

\section{ACKNOWLEDGMENT}

T. Tan would like to thank all the people in Backman's Biophotonics Lab at NU for providing him with a great experience in learning cutting-edge research in optics and biomedical cancer technology. In particular, many private discussions with I. Capoglu, L. Cherkezyan, S. Norris, P. Pradhan, A. Radosevich, J. Rogers, H. Subramanian and J. Yi have been immensely helpful to him in understanding wave propagations in biological cell phenomena.

\section{REFERENCES}

[1] A. Ajayi, "Direct Computation of Statistical Variations in Electromagnetic Problems," Doctor of Philosophy, Electrical and Electronic Engineering, University of Nottingham, U.K., 2008.

[2] A. Ajayi, P. Ingrey, P. Swwell, and C. Christopoulos, "Direct computation of statistical variations in electromagnetic problems," IEEE Trans. Electromagn. Compat., vol. 50, no. 2, pp. 325-332, May 2008.

[3] S. M. Smith, "Stochastic Finite Difference Time Domain," Doctor of Philosophy, Electrical and Computer Engineering, Univ. Utah, Provo, 2011.

[4] S. M. Smith and C. Furse, "A stochastic FDTD method for statistically varying biological tissues," in Proc. IEEE Int. Symp. APS/URSI, Spokane, WA, Jul. 2011, pp. 2274-2277.

[5] N. G. Van Kampen, Stochastic Processes in Physics and Chemistry. The Netherlands: North-Holland Personal Library, 2007.

[6] C. W. Gardiner, "Handbook of stochastic methods: For physics, chemistry and the natural sciences," in Springer Series in Synergetics. Berlin: Springer, 2010.

[7] L. R. A. X. de Menezes, A. Ajayi, C. Christopoulos, P. Sewewll, and G. A. Borges, "Efficient computation of stochastic electromagnetic problems using unscented transforms," IET Sci., Meas. Technol., vol. 2, no. 2, pp. 88-95, Mar. 2008.

[8] M. Scott, Applied Stochastic Processes in Science and Engineering, Chemistry and the Natural Sciences.

[9] K. Yee, "Numerical solution of initial boundary value problems involving Maxwell's equations in isotropic media," IEEE Trans. Antennas Propag., vol. 14, no. 3, pp. 302-307, May 1966.

[10] A. Taflove and M. E. Brodwin, "Numerical solution of steady-state electromagnetic scattering problems using the time-dependent Maxwell's equations," IEEE Trans. Microwave Theory Tech., vol. 23, no. 8, pp. 623-630, Aug. 1975.

[11] K. R. Umashankar and A. Taflove, "A novel method to analyze electromagnetic scattering of complex objects," IEEE Trans. Electromagn. Compat., vol. 24, pp. 397-405, 1982.

[12] A. Taflove and S. Hagness, Computational Electrodynamics: The Finite-Difference Time-Domain Method, 3rd ed. Boston, MA: Artech House, 2005.

[13] J. D. Jackson, Classical Electrodynamics, 3rd ed. Louisville: Hamilton Printing Co., 1999.

[14] J. A. Stratton, Electromagnetic Theory. New York: McGraw-Hill, 1941.

[15] R. F. Harrington, Time-Harmonic Electromagnetic Fields. New York: Wiley, 2001

[16] C. A. Balanis, Advanced Engineering Electromagnetics. New York: Wiley, 1989.

[17] E. Wolf, Principles of Optics, 5th ed. New York: Pergamon Press, 1975.

[18] H. Subramanian, P. Pradhan, Y. Liu, I. R. Capoglu, J. D. Rogers, H. K. Roy, R. E. Brand, and V. Backman, "Partial-wave microscopic spectroscopy detects subwavelength refractive index fluctuations: An application to cancer diagnosis," Opt. Lett., vol. 34, pp. 518-520, 2009.

[19] H. Subramanian, P. Pradhan, Y. Liu, I. R. Capoglu, X. Li, J. D. Rogers, A. Heifetz, D. Kunte, H. K. Roy, A. Taflove, and V. Backman, "Optical methodology for detecting histologically unapparent nanoscale consequences of genetic alterations in biological cells," $P N A S$, vol. 105, pp. 20118-20123, 2008.

[20] J. D. Rogers, I. R. Capoglu, A. Taflove, and V. Backman, "Accuracy of the born approximation in calculating the scattering coefficient of biological continuous random media," Opt. Lett., vol. 34, no. 17, Sep. 2009.

[21] A. Elsherbeni and V. Demir, The Finite-Difference Time-Domain Method for Electromagnetics With MATLAB Simulations. Herndon, VA: SciTech, 2009.

[22] J. B. Schneider, "Plane waves in FDTD simulations and a nearly perfect total-field/scattered-field boundary," IEEE Trans. Antennas Propag., vol. 52, no. 12, pp. 3280-3287, Dec. 2004. 
[23] T. Tan and M. E. Potter, "Optimized analytic field propagator (O-AFP) for plane wave injection in FDTD simulations," IEEE Trans. Antennas Propag., vol. 58, no. 3, pp. 824-831, Mar. 2010.

[24] T. Tan and M. E. Potter, "1-D multipoint auxiliary source propagator for the total-field/scattered-field FDTD formulation," IEEE Antennas Wireless Propag. Lett., vol. 6, pp. 144-148, 2007.

[25] T. Tan and M. E. Potter, "FDTD discrete planewave (FDTD-DPW) formulation for a perfectly matched source in TFSF simulations," IEEE Trans. Antennas Propag., vol. 58, no. 8, pp. 2641-2648, Aug. 2010.

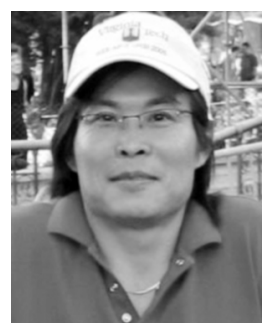

Tengmeng Tan (M'10) received the B.Sc. degree in electrical and computing engineering with a minor equivalence in applied mathematics and physics from University of Calgary, Canada, where he also completed the Ph.D. degree.

Prior to the graduate program, he was with IDENTEC Solution Inc. working as a Functional Test Engineer for the active RFID tags and later with Agilent Technology Inc. as a Board Level Design Engineer for the router tester project. His current research interests include both theoretical and practical aspects of wave propagation problems.

Mr. Tan was awarded an Excellent Teaching Assistant Award in 2005, and twice was a recipient of Student Paper Competition at the AP-S/URSI in 2008 and 2009, and University of Calgary Graduate Student High Productivity Achievement Award in 2010. He was also a recipient for the $2011 \mathrm{~S}$. A. Schelkunoff Transactions Prize Paper Award in recognition of the 2009 best paper published in the IEEE Transactions on Antennas and Propagation. He held an NSERC (Natural Sciences and Engineering Research Council of Canada) Postdoctoral Fellowship working as a Research Associate in Biomedical Engineering Department at Northwestern University, IL, collaborating with Allen Taflove and Vadim Backman.

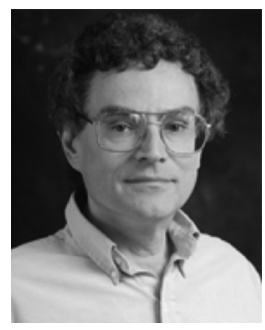

Allen Taflove (F'90) is a Professor of electrical engineering and computer science at Northwestern University, Evanston, IL. Since 1972, he has developed fundamental theoretical approaches, algorithms, and applications of finite-difference time-domain (FDTD) computational solutions of Maxwell's equations. He coined the descriptors "finite difference time domain" and "FDTD" in a 1980 IEEE paper, and in 1990 was the first person to be named an IEEE Fellow in the FDTD technical area. In 2002 , he was named by the Institute of Scientific
Information (ISI) to its original listing of the most-cited researchers worldwide, as published in ISIHighlyCited.com. According to Google Scholar, to-date his publications have received more than 20,000 citations, and the exact phrase "finite difference time domain" has appeared in over 48,000 articles. According to Microsoft Academic Search, the total citations of the first and second editions of his book, Computational Electrodynamics: The Finite Difference TimeDomain Method, rank 10th on its all-time list of the most-cited publications in all branches of engineering (of more than 3.3 million), and 5th all-time when considering primarily electrical engineering publications. In May 2010, Nature Milestones: Photons recognized him as one of the two principal pioneers of numerical methods for solving Maxwell's equations. Currently, continuing a collaboration that began in 2003, he is working with Prof. Vadim Backman of Northwestern's Biomedical Engineering Department. This research is aimed at the minimally invasive detection of early-stage cancers of the colon, pancreas, and lung. The techniques being pursued are based upon spectral and/or angular analysis of light that is backscattered from histologically normal tissue located away from a neoplastic lesion in what has been termed the field effect. This may lead to a new paradigm in cancer screening where, for example, lung cancer could be reliably detected by analyzing a few cells brushed from the interior surface of a person's cheek.

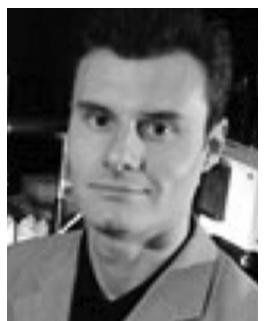

Vadim Backman received the Ph.D. degree in medical engineering from Harvard University and Massachusetts Institute of Technology.

$\mathrm{He}$ is a Professor of Biomedical Engineering at the McCormick School of Engineering and Applied Sciences, Northwestern University and a Program Leader, Cancer and Physical Sciences, at the Robert H. Lurie Comprehensive Cancer Center. His research is focused on bridging advances in biophotonics into biomedical research and medicine. He develops novel biophotonics technologies for characterization and imaging of biological tissue with a focus on the nanoscale, microscale and molecular levels. His research spans from technology development to cancer biophysics to large-scale clinical trials. He has authored over 130 journal publications, nine books and book chapters and 17 patents. In the past three years, he has served as the principle investigator on 16 grants from the National Institutes of Health and National Science Foundation. He is the Director of an NIH-funded Bioengineering Research Partnership.

Dr. Backman has received numerous awards including being selected as one of the top 100 young innovators in the world by MIT Technology Review Magazine and a Fellow of the American Institute for Medical and Biological Engineering. 